\title{
Object reconstruction from adaptive compressive measurements in feature-specific imaging
}

\author{
Jun Ke, ${ }^{1, \star}$ Amit Ashok, ${ }^{1}$ and Mark A. Neifeld ${ }^{1,2}$ \\ ${ }^{1}$ Department of Electrical and Computer Engineering, University of Arizona, Tucson, Arizona 85721, USA \\ ${ }^{2}$ College of Optical Science, University of Arizona, Tucson, Arizona 85721, USA \\ *Corresponding author: jke@ece.arizona.edu
}

Received 12 May 2010; accepted 30 July 2010;

posted 17 September 2010 (Doc. ID 128333); published 19 October 2010

\begin{abstract}
Static feature-specific imaging (SFSI), where the measurement basis remains fixed/static during the data measurement process, has been shown to be superior to conventional imaging for reconstruction tasks. Here, we describe an adaptive approach that utilizes past measurements to inform the choice of measurement basis for future measurements in an FSI system, with the goal of maximizing the reconstruction fidelity while employing the fewest measurements. An algorithm to implement this adaptive approach is developed for FSI systems, and the resulting systems are referred to as adaptive FSI (AFSI) systems. A simulation study is used to analyze the performance of the AFSI system for two choices of measurement basis: principal component (PC) and Hadamard. Here, the root mean squared error (RMSE) metric is employed to quantify the reconstruction fidelity. We observe that an AFSI system achieves as much as 30\% lower RMSE compared to an SFSI system. The performance improvement of the AFSI systems is verified using an experimental setup employed using a digital micromirror device (DMD) array. (C) 2010 Optical Society of America

OCIS codes: $\quad 100.0100,110.1758,110.1085,110.3010$.
\end{abstract}

\section{Introduction}

Researchers in the area of computational imaging have made significant advances toward addressing various important inverse problems, such as superresolution [1-3], point spread function engineering for estimation and recognition tasks [4-6], compressive imaging [7-9], and quantitative imaging based on controlled incident fields and amplitude-only scattered field measurements [10-12]. Here, we consider the problem of measurement basis design for compressive imaging systems using an adaptive approach.

Traditional imaging systems are designed with a goal of making an isomorphic measurement of a scene using image-formation optics and opto-electronic detector arrays, such as CCD/CMOS image sensors. However, this design approach ignores the fact that

0003-6935/10/340H27-13\$15.00/0

(C) 2010 Optical Society of America most natural scenes are inherently redundant and, therefore, sparse in some transform domains, such as Fourier and/or wavelets. This implies that by employing a suitable measurement basis, a scene/object can be directly measured in the sparse domain with fewer measurements. This provides the motivation for feature-specific imaging (FSI) [7], which employs sparse transforms, such as principal component (PC), Hadamard [13], and wavelets as the measurement basis. The resulting measurements are referred to as features, where each feature is an inner product between the object and a basis/projection vector. As a result of making fewer photon efficient measurements compared to conventional imaging, this approach results in system benefits, such as improved measurement signal to noise ratio (SNR) and reduced power, bandwidth, and weight requirements. As the number of measurements in FSI are typically much smaller than the object dimensionality, it is also sometimes referred to as "compressive imaging" [14-18]. The superior performance of FSI relative to conventional 
imaging has been demonstrated for various applications, such as sensor network, structured illumination, and face detection [19-22].

Previous research on FSI systems $[19,20,23]$ has employed measurement basis, such as the principal component or Karhunen-Loève basis, that incorporates the second-order correlation properties of natural scenes. It is important to emphasize that the measurement basis remains fixed or static during the measurement process. Therefore, we refer to such a system as a static FSI (SFSI) system. Note that while such a static measurement basis can be optimized to minimize the reconstruction mean square error (MSE) for an ensemble of objects; however, for a given object it becomes suboptimal. An adaptive approach would suggest modifying the measurement basis based on the specific object information derived from the previous measurements. This would allow an FSI system to adapt to the statistics of the specific object, thereby improving the overall system performance compared to an SFSI system. Here, we describe such an adaptive FSI (AFSI) system that uses PC and Hadamard bases as examples to illustrate the adaptive advantage. As mentioned earlier, the PC basis incorporates second-order object prior knowledge and is optimal in absence of measurement noise. The Hadamard basis does not incorporate such object knowledge, but it is commonly used in image processing. The remainder of the paper is organized into six sections. In Section 2, we review an SFSI system with PC and Hadamard projections. Then, we describe the AFSI system design in Section 3. This includes the description of algorithms using the two projections for both noise-free and noisy cases. In Section $\underline{4}$, a simulation study is used to quantify the performance of AFSI and SFSI systems. In Section 5, we describe the results from an experimental implementation of AFSI and SFSI systems that validates our simulation study. Finally, Section 6 summarizes the results and draws conclusions based on the reported simulation and experimental results.

\section{SFSI System Framework}

As mentioned earlier, an SFSI system uses projection vectors from a fixed/static measurement basis to make feature measurements. Here, we will begin with the noise-free case. Figure 1 shows a block diagram representation of an SFSI system. A lens array forms multiple images of an object. In the focal plane, an array of spatial light modulators (SLM) displaying the projection vectors modulates these images. Following the SLM array, each modulated image is spatially integrated onto a single detector, resulting in one feature measurement. An array $L$, such projections depicted in Fig. 1 yield $L$ feature measurements in parallel. After $I^{-}$such measurements, $M=I L$ features are collected. Mathematically, these measurement processes can be represented as $\mathbf{y}=\mathbf{F x}$, where $\mathbf{y}$ is the feature measurement vector of size $M \times 1, \mathbf{F}$ is the projection matrix of size $M \times N$, and $\mathbf{x}$ is the object vector of size $N \times 1$ obtained by lexicographically arranging a 2D object of size $\sqrt{N} \times \sqrt{N}$. In a PC-based SFSI (PC-SFSI) system, each row of $\mathbf{F}$ represents a projection vector that corresponds to an eigenvector of the object autocorrelation matrix $\mathbf{R}_{x}$. To minimize reconstruction error, the $M$ eigenvectors corresponding to the largest $M$ eigenvalues used to construct the PC projection matrix, yielding $M$ feature measurements [24]. The reconstruction process using noise-free measurements is represented as $\hat{\mathbf{x}}=\mathbf{F}^{T} \mathbf{y}$, where $\hat{\mathbf{x}}$ is the reconstructed object vector of size $N \times 1$. It is known that PC projections are optimal in terms of minimizing reconstruction MSE for an ensemble of objects assuming an underlying Gaussian distribution [24]. However, to minimize the reconstruction MSE with a minimum number of features for a specific object as opposed to an ensemble, the PC projection vectors need to be derived specifically for that particular object. For example, to reconstruct/estimate a "tank" object, a set of $M \mathrm{PC}$ projection vectors derived from tank training images generate a smaller error relative to another set of $M$ PC projection vectors derived from an ensemble of face images. Note that the first set of projection vectors derived from a training set composed of objects similar to the test object is able to incorporate more relevant object prior knowledge compared to a training set composed of a different class of objects than the test object. This observation motivates the design for our PC-based AFSI system discussed in the next section.

In a Hadamard-based SFSI (H-SFSI) system, the construction of the projection vectors does not depend on the object ensemble statistics. Figure 2(a) shows the first five Hadamard vectors using Sylvester's method for construction [13]. We define these vectors as Hadamard vectors sorted in Sylvester

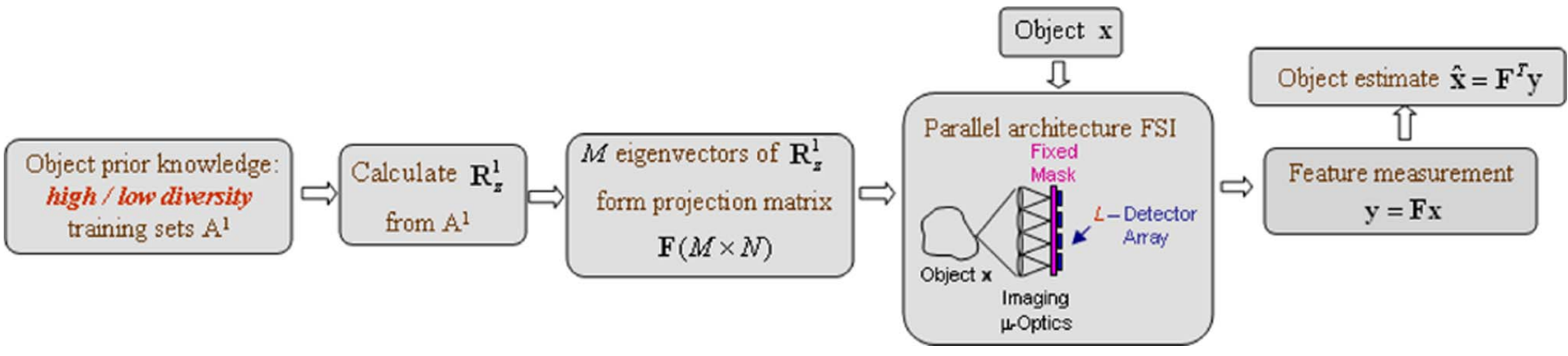

Fig. 1. (Color online) Block diagram for SFSI using PC bases. 

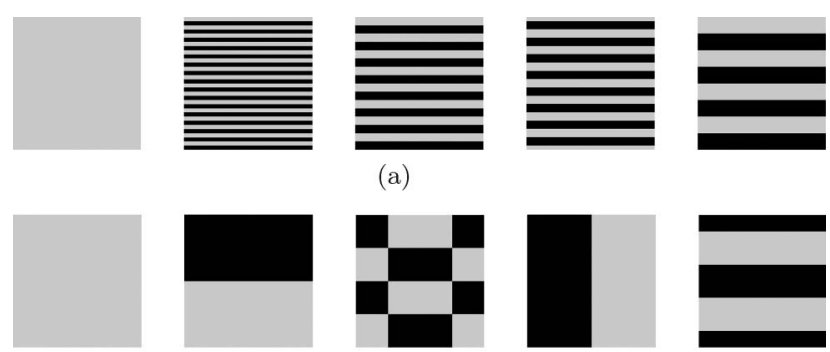

(a)
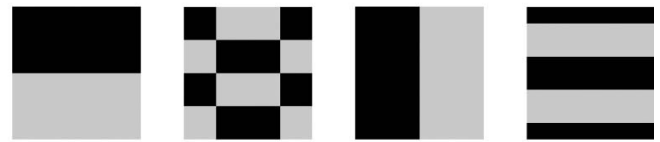

(b)
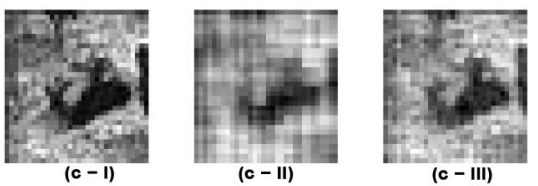

(c)

Fig. 2. Hadamard bases (a) sorted in Sylvester's order, (b) sorted in energy collection order, (c-I) object example; object reconstruction example using 200 Hadamard bases (c-II) sorted in Sylvester's order, (c-III) sorted in energy collection order.

order. Note that each feature measurement is an inner product between the corresponding projection vector and the object vector. Therefore, a different amount of object energy is collected in a feature using different Hadamard projection vectors. Sorting projection vectors by their energy collection efficiency results in ordering that we will refer to as the energy collection order. Figure 2(b) shows the first five Hadamard vectors in energy collection order for the $32 \times 32$ object shown in Fig. 2(c)-I. The reconstructions obtained by using 200 Hadamard vectors sorted using both the orders are shown in Figs. 2(c)-II and c-III, respectively. Observe that the reconstruction in Fig. 2(c)-III is superior to that in Fig. 2(c)-II. Therefore, from Fig. 2, we conclude that using the energy collection ordering of Hadamard projection vectors improves the reconstruction performance. Here, we employ a set of training objects to sort the Hadamard projection vectors according to the energy collection order for use in the H-SFSI system.

We will use a parallel architecture for a featurespecific (FS) imager to describe the system implementation in the following discussion. In such an FS imager, $L$ features are measured in parallel during each time period $T_{0}$. One way to implement a parallel architecture FS imager is to use a lenslet array with $L$ elements as shown in Fig. 1 . The object irradiance in each feature measurement is reduced by a factor $L$ due to use of $L$ lenslets of equal diameter within the system aperture [23]. The detector noise in the FS imager is assumed to be independent additive white Gaussian noise with zero mean. Its variance is $\sigma^{2}=\sigma_{0}^{2} / T_{0}$, where $\sigma_{0}^{2}$ is the noise energy per bandwidth and $T_{0}$ is the detector exposure time [23].

Including detector noise, $M=I L$ feature measurements in a SFSI system are expressed as

$$
\mathbf{y}=\mathbf{F x}+\mathbf{n},
$$

where $\mathbf{F}$ is the projection matrix of size $M \times N$, which includes the $1 / L$ factor resulting from the choice of the parallel architecture, and $\mathbf{n}$ is the noise vector of size $M \times 1$. The object estimate $\hat{\mathbf{x}}$ can be obtained using a Wiener or a linear minimum mean square error (LMMSE) operator $\mathbf{W}$ as $\hat{\mathbf{x}}=\mathbf{W y}$, where

$$
\mathbf{W}=\mathbf{R}_{x} \mathbf{F}^{T}\left[\mathbf{F} \mathbf{R}_{x} \mathbf{F}^{T}+\mathbf{R}_{n}\right]^{-1}
$$

and $\mathbf{R}_{n}$ is the noise autocorrelation matrix, $\mathbf{R}_{n}=\sigma^{2} \mathbf{I}$.

\section{AFSI System Framework}

In an AFSI system, the measurement basis is updated based on the current and past measurements. For example, for a $\sqrt{N} \times \sqrt{N}$ object, we design the first $L$ projection vectors, then use them to make the corresponding $L$ feature measurements. Based on these $L$ measurements, we design the second $L$ projections and make the second $L$ feature measurements and so on. Following this procedure, we design projection vectors sequentially incorporating object knowledge learned at each step. In this section, we describe the AFSI system design for two cases: 1) in presence and 2) in the absence of noise. Before continuing the discussion, we need to define a list of parameters as shown below:

x: object vector of size $N \times 1$.

$A^{(i)}, B^{(i)}$ : two training sets in the $i$ th iteration, where samples in $A^{(i)}$ span a $N-i L$ dimensional space and samples in $B^{(i)}$ span a $N$ dimensional space.

$k(i)$ : cardinality of $A^{(i)}$ and $B^{(i)}$.

$\mathbf{R}_{A}^{(i)}, \mathbf{R}_{B}^{(i)}$ : autocorrelation matrices estimated from samples in $A^{(i)}$ and $B^{(i)}$.

$\mathbf{F}^{(i)}$ : projection matrix of size $L \times N$ in the $i$ th iteration.

$\tilde{\mathbf{F}}^{(i)}$ : accumulated projection matrix of size $(i L) \times N$ after the first $i$ iterations.

$\mathbf{n}^{(i)}$ : noise vector of size $L \times 1$ in the $i$ th iteration.

$\mathbf{y}^{(i)}$ : feature vector of size $L \times 1$ in the $i$ th iteration.

$\tilde{\mathbf{y}}^{(i)}$ : accumulated feature vector of size $(i L) \times 1$ after the first $i$ iterations.

$\mathbf{W}^{(i)}$ : reconstruction operator in the $i$ th iteration when there is no noise in measurement.

$\mathbf{W}_{n}^{(i)}$ : reconstruction operator in the $i$ th iteration when there is noise in measurement.

$\hat{\mathbf{x}}^{(i)}$ : object reconstruction in the $i$ th iteration.

\section{A. AFSI System in the Absence of Noise}

\section{PC-Based AFSI (PC-AFSI) System}

Figure 3 presents the block diagram of a PC-AFSI system. In each adaptation iteration, $L$ PC projection vectors are derived from a training set $A^{(i)}$. The vectors are the $L$ dominant eigenvectors of $\mathbf{R}_{A}^{(i)}$, where $i$ denotes the $i$ th iteration, $i=1,2,3, \ldots, I$, and $I$ is the total number of adaptation steps. Feature measurements in the $i$ th iteration are represented as $\mathbf{y}^{(i)}=$ $\mathbf{F}^{(i)} \mathbf{x}$. All of the measurements up to the $i$ th iteration are represented as $\tilde{\mathbf{y}}^{(i)}=\left[\mathbf{y}^{(1) T} \mathbf{y}^{(2) T} \ldots \mathbf{y}^{(i) T}\right]^{T}$. To include the object prior knowledge, $A^{(i)}$ is updated to 


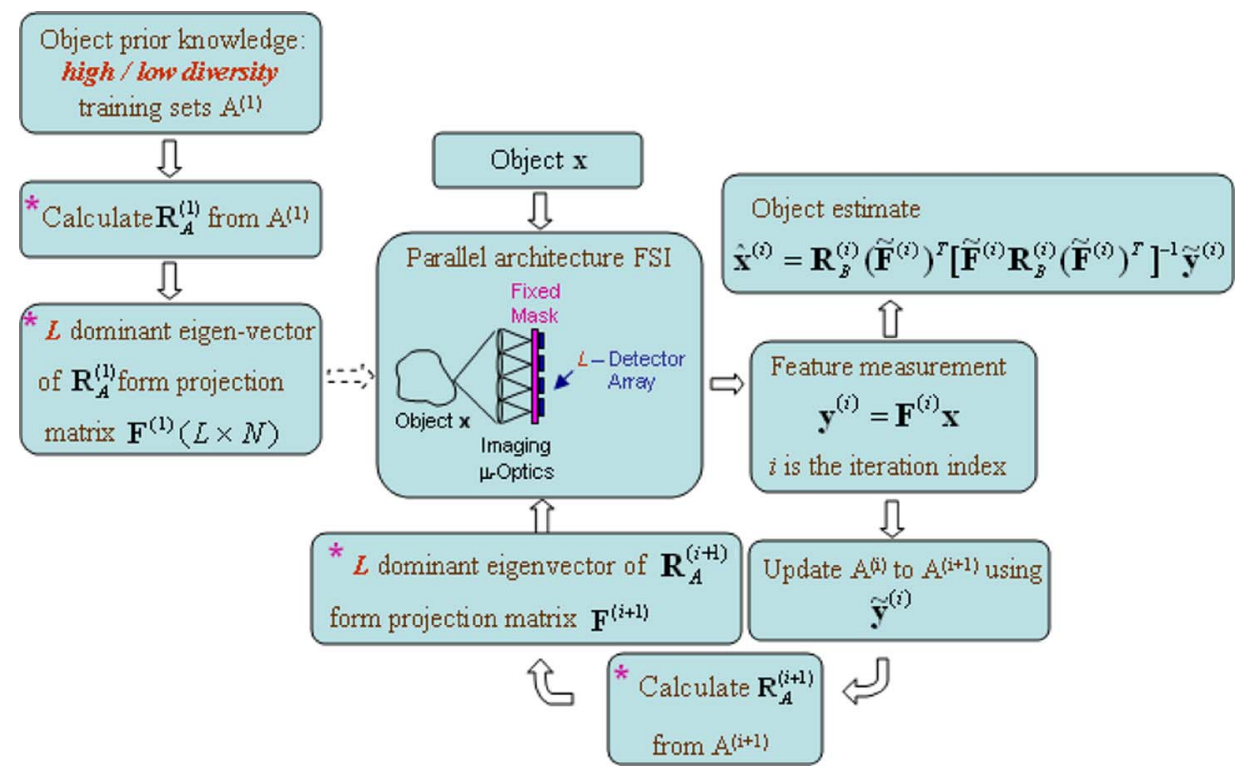

Fig. 3. (Color online) Block diagram for AFSI using a PC basis, where * indicates the blocks distinguishing FASI using a PC basis from those using a Hadamard basis.

$A^{(i+1)}$ using the " $K$-nearest neighbor" method in the accumulated feature space. In such a method, the $k(i+1)$ closest training samples to $\hat{\mathbf{x}}^{(i)}$ are chosen from $A^{(i)}$ to form $A^{(i+1)}$, and the rest of the samples are discarded. To ensure the orthogonality among all projections, we remove the subspace spanned by the $i L \mathrm{PC}$ projections from $A^{(i+1)}$. The number of training samples $k(i)$ is a function of $i$, which exponentially decrease as $i$ increases, $k(i)=\alpha e^{-\beta i}$. Parameters $\alpha$ and $\beta$ are searched using more than 50 training samples. The $\alpha$ and $\beta$ values optimizing AFSI system performance are used throughout the simulation and experiment in this paper.

With $i L$ feature measurements, the object estimation is $\hat{\mathbf{x}}^{(i)}=\mathbf{W}^{(i)} \tilde{\mathbf{y}}(i)$, where $\mathbf{W}^{(i)}=\mathbf{R}_{B}^{(i)} \tilde{\mathbf{F}}^{(i) T} \times$ $\left[\tilde{\mathbf{F}}^{(i)} \mathbf{R}_{B}^{(i)} \tilde{\mathbf{F}}^{(i) T}\right]^{-1}$. $\tilde{\mathbf{F}}^{(i)}$ is the accumulated projection matrix, $\tilde{\mathbf{F}}^{(i)}=\left[\begin{array}{llll}\mathbf{F}^{(1) T} & \mathbf{F}^{(2) T} & \ldots & \mathbf{F}^{(i) T}\end{array}\right]^{T} . \mathbf{R}_{B}^{(i)}$ is derived from the sample set $B^{(i)}$ with $B^{(1)}=A^{(1)}$ initially. $B^{(i)}$ is also updated using the same " $K$-nearest neighbor" as $A^{(i)}$. However, the subspace spanned by the $i L$ projections is not removed from $B^{(i+1)}$ in order to make sure that $\tilde{\mathbf{F}}^{(i)} \mathbf{R}_{B}^{(i)} \tilde{\mathbf{F}}^{(i) T}$ is invertible.

The following is the algorithm for estimating the object $\mathbf{x}$ using $I$ iterations in a PC-AFSI system.

1. Calculate $\mathbf{R}_{A}^{(i)}=E\left\{\mathbf{a}_{j}^{(i)} \mathbf{a}_{j}^{(i) T}\right\}$ and $\mathbf{R}_{B}^{(i)}=E\left\{\mathbf{b}_{j}^{(i)} \mathbf{b}_{j}^{(i) T}\right\}$, where $E\{\}$ represents the mathematical expectation, $\mathbf{a}_{j}^{(i)}$ and $\mathbf{b}_{j}^{(i)}$ are training samples in $A^{(i)}$ and $B^{(i)}$, respectively, and $j=1,2, \cdots, k(i)$. Initially, $A^{(1)}=B^{(1)}$ and $k(1)=\alpha e^{-\beta}$.

2. Calculate the $L$ dominant eigenvectors of $\mathbf{R}_{A}^{(i)}$ and form $\mathbf{F}^{(i)}$.

3. Make the feature measurements $\mathbf{y}^{(i)}=\mathbf{F}^{(i)} \mathbf{x}$ and form $\tilde{\mathbf{y}}^{(i)}=\left[\begin{array}{llll}\mathbf{y}^{(1) T} & \mathbf{y}^{(2) T} & \cdots & \mathbf{y}^{(i) T}\end{array}\right]^{T}$.

4. Estimate the object $\hat{\mathbf{x}}^{(i)}=\mathbf{W}^{(i)} \tilde{\mathbf{y}}^{(i)}$.

5. Calculate the feature measurements of $\mathbf{a}_{j}^{(i)}, \mathbf{u}_{j}^{(i)}=\mathbf{F}^{(i)} \mathbf{a}_{j}^{(i)}$. Form $\tilde{\mathbf{u}}_{j}^{(i)}=\left[\begin{array}{llll}\mathbf{u}_{j}^{(1) T} & \mathbf{u}_{j}^{(2) T} & \cdots & \mathbf{u}_{j}^{(i) T}\end{array}\right]^{T}$.

6. Calculate the distance between $\hat{\mathbf{x}}^{(i)}$ and $\mathbf{a}_{j}^{(i)}$ in feature space: $d_{j}^{(i)}=\left\|\tilde{\mathbf{u}}_{j}^{(i)}-\tilde{\mathbf{y}}^{(i)}\right\|_{2}$.
7. Update $A^{(i)}$ to $A^{(i+1)}$ by choosing $\mathbf{a}_{j}^{(i)}$ such that $\mathbf{a}_{j}^{(i)}$ is associated with the $k(i+1)$ smallest $d_{j}^{(i)}$ values. $B^{(i)}$ is also updated to $B^{(i+1)}$ accordingly.

8. Define the samples in $A^{(i+1)}$ as $\mathbf{a}_{j}^{(i+1)}=\mathbf{a}_{j}^{(i)}-\mathbf{F}^{(i) T} \mathbf{u}_{j}^{(i)}$.

9. If $i<I$, go back to 1 .

\section{Hadamard-Based AFSI (H-AFSI) System}

The feature adaptation procedure for a H-AFSI system is a slightly modified version of the PC-AFSI system. In the H-AFSI system, the projection vectors are fixed and independent of the training set. However, the implementation order of the vectors can be adapted using training samples. We use the training sample average of $A^{(i)}$ to choose the $L$ dominant vectors in the sense of the object energy collection. For example, if $\overline{\mathbf{x}}$ is the training sample average vector, and $\left|\overline{\mathbf{x}}^{T} \mathbf{f}_{1}\right|>\left|\overline{\mathbf{x}}^{T} \mathbf{f}_{2}\right|>\cdots>\left|\overline{\mathbf{x}}^{T} \mathbf{f}_{L}\right|>\cdots>\left|\overline{\mathbf{x}}^{T} \mathbf{f}_{N}\right|$ where $\mathbf{f}_{i},(i=1,2, \cdots, L \cdots, N)$ are a set of Hadamard vectors, then $\mathbf{f}_{1}, \mathbf{f}_{2}, \cdots$, and $\mathbf{f}_{L}$ are the chosen vectors. $A^{(i)}$ is updated in the same manner as in the PC-AFSI system, specifically using the PC-AFSI algorithm steps 5-8. Once again, the object estimate in the $i$ th iteration is $\hat{\mathbf{x}}^{(i)}=\mathbf{W}^{(i)} \tilde{\mathbf{y}}^{(i)}$. The block diagram and the algorithm in a H-AFSI system is similar to that of a PC-AFSI system, except that the diagram blocks marked with star in Fig. 3 and the algorithm steps 1 and 2 for the PC-AFSI system need to be modified. Instead of the $L$ dominant eigenvectors of $\mathbf{R}_{A}^{(i+1)}$, the $L$ dominant Hadamard vectors in the sense of object energy collection are chosen to form projection matrix $\mathbf{F}^{(i+1)}$.

\section{B. AFSI System in the Presence of Noise}

So far, we have considered a noise-free feature measurement model. Now, we extend our model to include additive detector noise. The AFSI system feature measurements in the $i$ th iteration can be expressed as $\mathbf{y}^{(i)}=\mathbf{F}^{(i)} \mathbf{x}+\mathbf{n}^{(i)}$. The object estimate 
is $\hat{\mathbf{x}}^{(i)}=\mathbf{W}_{n}^{(i)} \tilde{\mathbf{y}}^{(i)}$, where $\mathbf{W}_{n}^{(i)}$ is the Wiener/LMMSE operator $\mathbf{W}_{n}^{(i)}=\mathbf{R}_{B}^{(i)} \tilde{\mathbf{F}}^{(i) T}\left[\tilde{\mathbf{F}}^{(i)} \mathbf{R}_{B}^{(i)} \tilde{\mathbf{F}}^{(i) T}+\mathbf{R}_{n}\right]^{-1}$ and where $\mathbf{R}_{n}=\sigma^{2} \mathbf{I}$ is the noise autocorrelation matrix. The training sets are updated using denoised accumulated feature measurements. The denoised features are defined as

$$
\hat{\mathbf{y}}^{(i)}=\mathbf{W}_{y}^{(i)} \mathbf{y}^{(i)}
$$

where

$$
\mathbf{W}_{y}^{(i)}=\mathbf{R}_{y}^{(i)}\left[\mathbf{R}_{y}^{(i)}+\mathbf{R}_{n}\right]^{-1}
$$

and

$$
\begin{aligned}
\mathbf{R}_{y}^{(i)} & =E\left\{\left[\mathbf{F}^{(i)} \mathbf{x}\right]\left[\mathbf{F}^{(i)} \mathbf{x}\right]^{T}\right\} \\
& =\mathbf{F}^{(i)} \mathbf{R}_{B}^{(i)} \mathbf{F}^{(i) T}
\end{aligned}
$$

The following is the algorithm for an AFSI system collecting $M=I L$ features in the presence of noise:

1. Calculate $\mathbf{R}_{B}^{(i)}=E\left\{\mathbf{b}_{j}^{(i)} \mathbf{b}_{j}^{(i) T}\right\}$ and form $\mathbf{W}_{n}^{(i)}$, where $E\{\}$ represents the mathematical expectation, $\mathbf{b}_{j}^{(i)}$ are training samples in $B^{(i)}$, and $j=1,2, \cdots, k(i)$. Initialize $A^{(1)}=B^{(1)}$ and $k(1)=\alpha e^{-\beta}$.

2. Use $A^{(i)}$ to decide the $L$ most dominant projection vectors and form $\mathbf{F}^{(i)}$.

3. Make the feature measurements $\mathbf{y}^{(i)}=\mathbf{F}^{(i)} \mathbf{x}$ and form $\tilde{\mathbf{y}}^{(i)}=\left[\begin{array}{llll}\mathbf{y}^{(1) T} & \mathbf{y}^{(2) T} & \ldots & \mathbf{y}^{(i) T}\end{array}\right]^{T}$.

4. Estimate the object $\hat{\mathbf{x}}^{(i)}=\mathbf{W}_{n}^{(i)} \tilde{\mathbf{y}}^{(i)}$.

5. Calculate the denoised feature vector $\hat{\mathbf{y}}^{(i)}=\mathbf{W}_{y}^{(i)} \mathbf{y}^{(i)}$

6. Calculate the feature measurements of $\mathbf{a}_{j}^{(i)}, \mathbf{u}_{j}^{(i)}=\mathbf{F}^{(i)} \mathbf{a}_{j}^{(i)}$. Form $\tilde{\mathbf{u}}_{j}^{(i)}=\left[\begin{array}{llll}\mathbf{u}_{j}^{(1) T} & \mathbf{u}_{j}^{(2) T} & \ldots & \mathbf{u}_{j}^{(i) T}\end{array}\right]^{T}$.

7. Calculate the distance between $\hat{\mathbf{x}}^{(i)}$ and $\mathbf{a}_{j}^{(i)}$ in feature space: $d_{j}^{(i)}=\left\|\tilde{\mathbf{u}}_{j}^{(i)}-\tilde{\hat{\mathbf{y}}}^{(i)}\right\|_{2}$, where $\tilde{\hat{\mathbf{y}}}^{(i)}=\left[\begin{array}{lll}\hat{\mathbf{y}}^{(1) T} & \hat{\mathbf{y}}^{(2) T} \quad \ldots \quad \hat{\mathbf{y}}^{(i) T}\end{array}\right]^{T}$.

8. Update $A^{(i)}$ to $A^{(i+1)}$ by choosing $\mathbf{a}_{j}^{(i)}$ such that $\mathbf{a}_{j}^{(i)}$ is associated with the $k(i+1)$ smallest $d_{j}^{(i)}$ values. $B^{(i)}$ is also updated to $B^{(i+1)}$ accordingly.

9. Define the samples in $A^{(i+1)}$ as $\mathbf{a}_{j}^{(i+1)}=\mathbf{a}_{j}^{(i)}-\mathbf{F}^{(i) T} \mathbf{u}_{j}^{(i)}$.

10 . If $i<I$, go back to 1 .

\section{Simulation Results}

We use 97,392 samples of size $32 \times 32$, including face, building, and tank images obtained under high, moderate, and low illumination conditions for training. Two types of training sets are used in our simulations: (1) high-diversity and (2) low-diversity. The high-diversity training set $A_{H}$ includes all three types objects, and the low-diversity training set $A_{L}$ includes only one type of object. Tank object samples form the low-diversity training set in this case. Figures 4(a) and 4(b) show six training samples from high-diversity and low-diversity training sets, respectively. Twenty tank object images are used for testing. The reconstruction error is quantified using the relative RMSE metric, which is defined as $E\left\{\left\|\hat{\mathbf{x}}^{(i)}-\mathbf{x}\right\|_{2} /\|\mathbf{x}\|_{2}\right\} . E\{\}$ denotes the mathematical expectation over the testing set. To simplify notation, RMSE refers to relative RMSE throughout the rest of the paper.

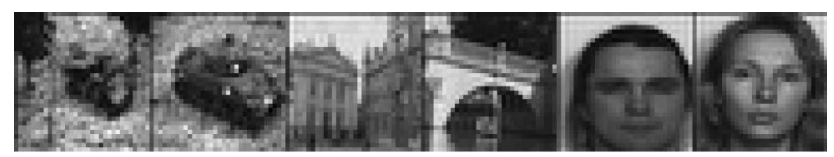

(a)

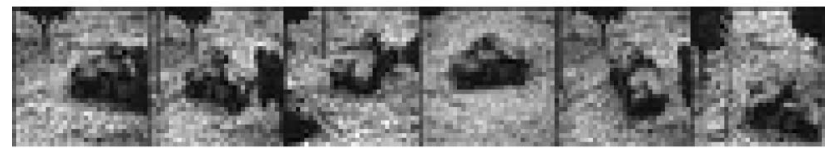

(b)

Fig. 4. Image samples from a (a) high- and (b) low-diversity training set.

\section{A. PC-SFSI and PC-AFSI Systems in the}

Absence of Noise

In Figs. 5(a) and 5(b) we plot reconstruction RMSE in an AFSI system as a function of the number of feature measurements, denoted by $M$, for training sets $A_{H}$ and $A_{L}$, respectively. We consider $L=1$ and $L=$ 10 cases for both training sets. We also present the reconstruction performance of the SFSI system with unknown and known objects. These two SFSI systems are labeled as "SFSI" and "SFSI-obj prior," respectively. With an unknown object, the PC projections are derived using all training samples. With the known object, the $N$ training samples closest to the object in the sense of Eucledian distance are chosen to estimate the object autocorrelation matrix and its eigenvectors for the projection design. Because these samples are the most similar samples to the object, the designed projection vectors make the reconstruction error smaller than the error using all other projection vectors designed from the same training set. From Figs. 5(a) and 5(b), we can make following observations. First, as evident from both figures, adaptation significantly improves the reconstruction performance. We use the high-diversity training set $A_{H}$ and $L=1$ as an example. For $M=250$ features, the RMSE is 0.150 in the SFSI system and 0.103 in the AFSI system, which is an improvement of RMSE $31.32 \%$. Similarly, for the low-diversity training set $A_{L}$, we observe a slightly smaller RMSE improvement $23.72 \%$. Note that the reconstruction error of SFSI system using $A_{L}$ is smaller than using $A_{H}$, because $A_{L}$ includes only tank samples and therefore has more object prior knowledge. However, reconstruction error in the AFSI system remains about the same, regardless of the training set diversity because the projection vectors adapt to specific objects in the testing sample. This is evident from the RMSE values for AFSI systems using $A_{H}$ and $A_{L}$ with $L=1$ are 0.103 and 0.102 , respectively. The second observation is that the AFSI system using different choices of $L$ has nearly the same reconstruction performance. Using $A_{H}$ with $M=250$, the RMSE is 0.101 for $L=10$ and 0.103 for $L=1$. The reason is that with a properly chosen training set update sequence $k(i)=0.5 e^{-0.0063 i}$, the AFSI performance is independent, with $L$ using noise-free measurements. Finally, we observe that the AFSI system performance is close to the performance upper bound 


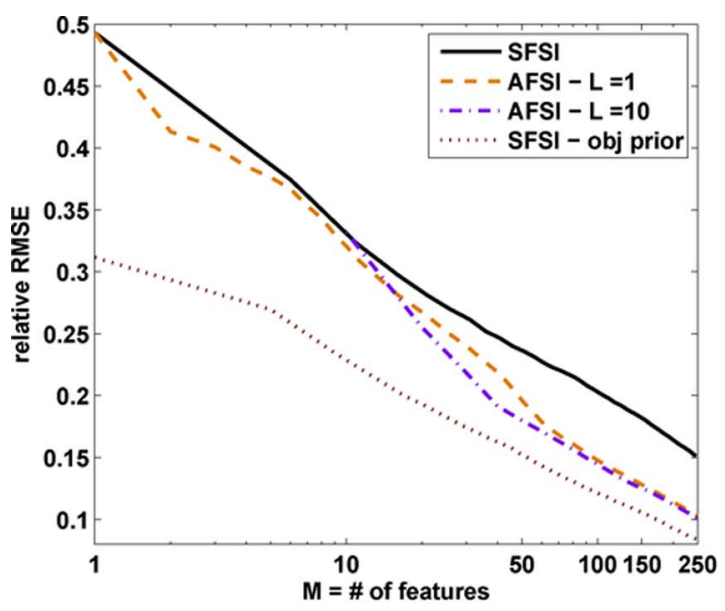

(a)

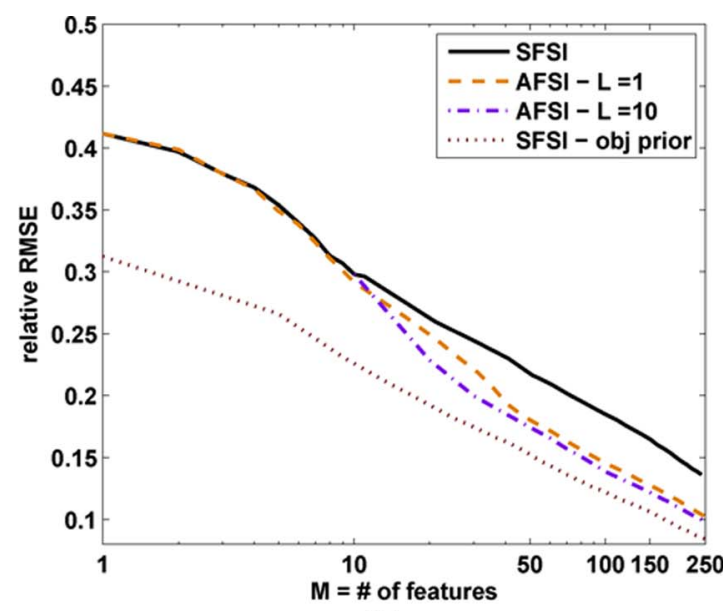

(b)

Fig. 5. (Color online) RMSE versus $M$ in PCA-based SFSI systems and AFSI systems for $L=1,10$, and 20 using (a) high- and (b) lowdiversity training sets.

defined by the "SFSI-obj prior" system. With $M=250$, the relative reconstruction error of the "SFSI-obj prior" system is 0.084 for $A_{H}$ compared with the RMSE value 0.101 in AFSI system. In Fig. $\underline{6}$, testing sample and reconstructions using $M=$ 100 and $M=260$ adaptive PC features are presented. The training set has high diversity. As expected, visually and quantitatively, the reconstructions using the AFSI system in Figs. 6(c) and 6(e) are better than the reconstructions in Figs. $\overline{6(b)}$ and 6(d) using the SFSI system. Table 1 summarizes the RMSE values for PC-based SFSI and AFSI systems using $A_{H}$ and $A_{L}$ training sets for $L=1,10$, and $M=250$.

\section{B. H-SFSI and H-AFSI Systems in the Absence of Noise}

We repeat our simulation study for H-SFSI and $\mathrm{H}-$ AFSI systems. Figure 7(a) presents a plot of RMSE versus $M$ for the H-AFSI system using training set $A_{H}, L=1$, and 10 . The results for the SFSI and "SFSI_obj prior" systems are also plotted. Similar to the PC-AFSI system, the H-AFSI system yields a superior reconstruction performance relative to the SFSI system. The RMSE improvements are $31 \%$ and $27.27 \%$ for $L=10$ and $L=1$, respectively, when $M$ is 250 . Once again, we observe that the reconstruction performance of the AFSI system is close to the performance of the "SFSI—obj prior" system.
We also study the SFSI and AFSI systems using lowdiversity training set $A_{L}$. We have similar observations as for the PC-AFSI and PC-SFSI systems using training set $A_{L}$. The results are plotted in Fig. 7(b). The RMSE resulted are tabulated in Table $\underline{2}$.

\section{AFSI Systems in the Presence of Noise}

Throughout the rest of this section, the detector noise energy per bandwidth in an FSI system is assumed to be $\sigma_{0}^{2}=1$, while the object samples have dynamic range of $[0,255]$. For detector exposure time $T_{0}$, the noise variance in feature measurement $\sigma^{2}=\sigma_{0}^{2} / T_{0}=$ $1 / T_{0}$. Figure 8 shows some object examples and their noisy measurements with conventional imaging when $\sigma^{2}=1 / T_{0}$ and $T_{0}=0.02 \mathrm{~s}$. In Fig. 9(a), we present the reconstruction error RMSE versus $M$ for a FSI system employing the parallel architecture with $L=4$ and $T_{0}=0.02 \mathrm{~s}$. From this figure, we can make the following observations. First, as $M$ increases, the reconstruction error reduces for all FSI systems while the PC-FSI systems achieve the minimum error. For both projections, there is a nonzero minimum RMSE value due to finite measurement SNR. Two components contribute to the reconstruction error: the truncation error and the feature measurement SNR. The truncation error occurs when using $M<N$ feature measurements; therefore, it decreases as $M$ increases toward $N$. As the number

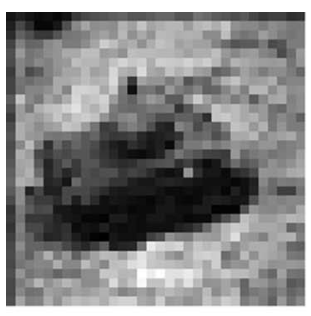

(a)

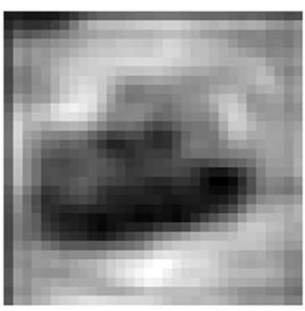

(b)

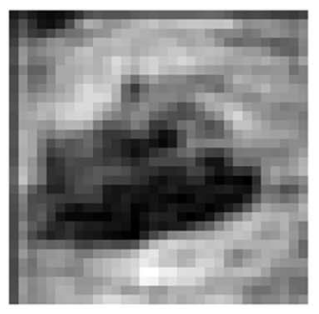

(c)

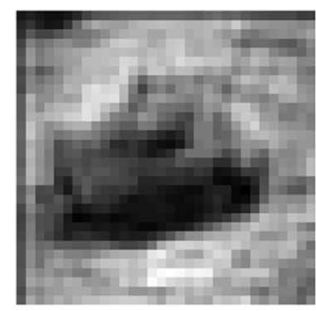

(d)

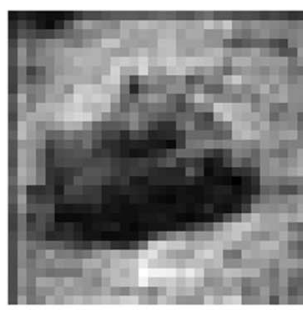

(e)

Fig. 6. Examples of (a) object reconstruction using a PCA-based system; (b) using an SFSI system for $M=100$, (c) using an SFSI system for $M=260$, (d) using an AFSI system for $M=100$, and (e) using an AFSI system for $M=260$ with a high-diversity training set $A_{H}$. 
Table 1. RMSE in PC-SFSI and PC-AFSI Systems for $L=1,10$, with $M=250$ Using High- and Low-Diversity Training Sets

\begin{tabular}{|c|c|c|c|c|}
\hline & \multicolumn{2}{|c|}{ High $A_{H}$} & \multicolumn{2}{|c|}{ Low $A_{H}$} \\
\hline & Relative RMSE & RMSE Improvement & Relative RMSE & RMSE Improvement \\
\hline SFSI & 0.150 & $\times$ & 0.134 & $\times$ \\
\hline AFSI $-L=10$ & 0.101 & $32.44 \%$ & 0.098 & $26.93 \%$ \\
\hline SFSI—obj prior & 0.084 & $44.07 \%$ & 0.084 & $37.28 \%$ \\
\hline
\end{tabular}

of measurement $M$ increases, the feature measurement SNR associated with the higher-order feature decreases. This trend can be understood by noting that the signal energy in each feature measurement decreases, both for PC features and energy-sorted Hadamard features, with increasing $M$ while the detector noise remains fixed. Thus, ultimately, the feature measurement SNR limits the minimum reconstruction error achievable, and it determines the number of features $M$ at which it is first achieved. The second observation from Fig. 9(a) is that both the PC-AFSI and H-AFSI systems have a smaller reconstruction RMSE than the corresponding SFSI systems. The third observation is that the PC-based systems have a smaller reconstruction RMSE than the Hadamard-based systems for small $M$. However, for large $M$, the PC-based systems have a larger RMSE than that of the Hadamard-based system. This is consistent with the observation described in Ref. [19], that Hadamard projection vectors have better photon throughput compared to the PC projection vector. This benefit becomes significant as $M$ increases and the feature energy corresponding to higher-order features is larger for Hadamard features compared to PC features, thus resulting in higher feature measurement SNR, given the detector noise remains fixed. Figure 9(b) presents the RMSE versus $M$ for a PC-based AFSI system with $L=4,16$, and 32 . Note that, while in the noise-free measurement case described in Section 3.A.1, the reconstruction RMSE remains relatively unchanged with increasing $L$, in the noisy measurements case the reconstruction RMSE increases with increasing $L$. This can be understood by noting that in a parallel architecture for an FSI system with $L$ measurements, while the detector noise variance is fixed as $1 / T_{0}$, the signal energy associated with each feature measurement reduces as $L$ increases. Therefore, the system reconstruction performance reduces as a result of decreasing feature measurement SNR with increasing $L$. Also note that the minimum reconstruction RMSE achieved is reached first at a lower $M$ as $L$ increases. This is due to the fact that the Wiener reconstruction operator effectively limits the use of feature measurement with low SNR $(<1)$ to minimize reconstruction error. Thus, as $L$ increases and feature measurement SNR drops with increasing $L$, the Wiener achieves the minimum error with fewer feature measurements. Figure 9(c) shows the RMSE versus $M$ plot for a Hadamard-based AFSI system with $L=4,16$, and 32 . Once again, reconstruction error increases as $L$ increases for the same reason as in the case of PC-FSI systems.

Figure 10 shows a plot of RMSE versus $M$ for different detector exposure time $T_{0}$ when $L=32$ in a PC-based AFSI system. Because the noise energy is inversely proportional to $T_{0}$, the AFSI system has a smaller reconstruction error for larger $T_{0}$ due to lower detector noise, hence, larger measurement SNR. Given an RMSE value, we can find the minimum number of features $M_{\min }$ and the total feature collection time $T_{\text {total }}=T_{0} * M_{\text {min }}$ required to reach

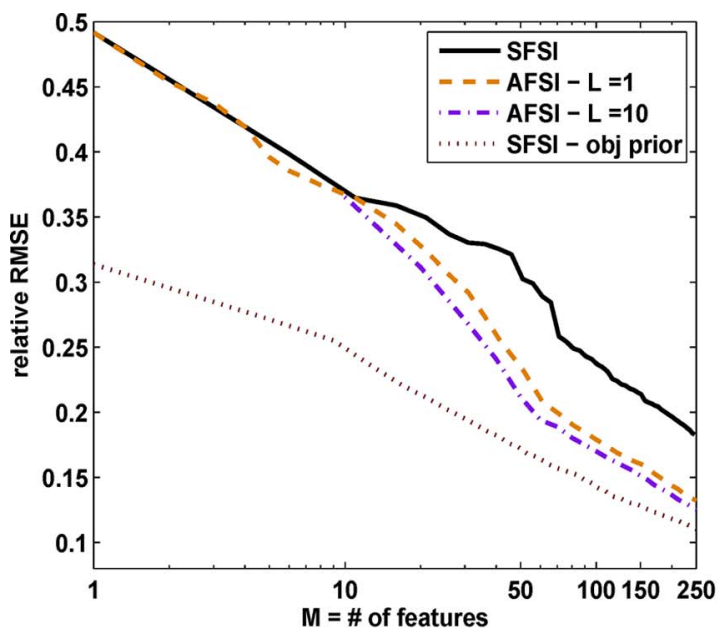

(a)

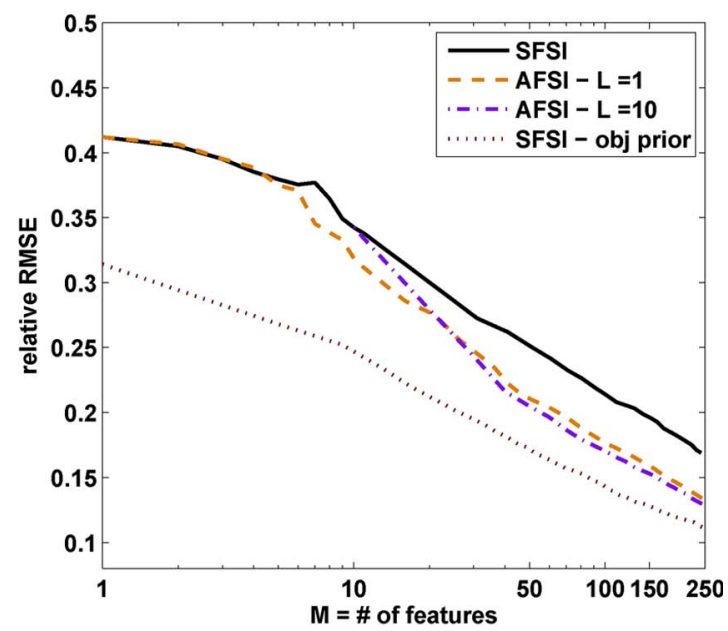

(b)

Fig. 7. (Color online) RMSE versus $M$ in an H-AFSI system for $L=1,10$ using (a) a high- and (b) low-diversity training sets. 
Table 2. RMSE in H-SFSI and H-AFSI Systems for $L=1,10$ with $M=250$ Using High- and Low-Diversity Training Sets

\begin{tabular}{|c|c|c|c|c|}
\hline & \multicolumn{2}{|c|}{ High $A_{H}$} & \multicolumn{2}{|c|}{ Low $A_{L}$} \\
\hline & Relative RMSE & RMSE Improvement & Relative RMSE & RMSE Improvement \\
\hline SFSI & 0.182 & $\times$ & 0.167 & $\times$ \\
\hline AFSI $-L=10$ & 0.126 & $30.95 \%$ & 0.128 & $23.40 \%$ \\
\hline SFSI—obj prior & 0.110 & $39.44 \%$ & 0.110 & $33.82 \%$ \\
\hline
\end{tabular}

a desired reconstruction error. Figs. 11(a)-11(c) show $T_{\text {total }}$ versus $T_{0}$ in PC-based SFSI and AFSI systems, respectively, with $L=1,4$, and 16, and RMSE values equal to 0.10 and 0.15 . The corresponding plots for the Hadamard-based SFSI and AFSI systems are presented in Figs. 11(d)-11(f), respectively. Two contributions determine $T_{\text {total }}$. When $T_{0}$ is large, the measurement noise is small. However, more time is required to acquire the data and the system loses its adaptation advantage. When $T_{0}$ is small, the measurement noise is large, but the system is more agile and can more quickly exploit knowledge acquired from measurements. Therefore, for each value of $L$, there exists a minimum $T_{\text {total }}$ where the measurement noise and adaptation balance out each other. We observe such minimum $T_{\text {total }}$ values in Figs. 11(b), 11(c), 11(e), and 11(f). Note that the system can not reach the RMSE requirement if $T_{0}$ is too small (i.e., if noise is too large). Therefore, some curves in Figs. 11 have their minimum $T_{\text {total }}$ values at the smallest $\overline{T_{0}}$ values. Another observation is that the $T_{\text {total }}$ in an AFSI system is always smaller than the $T_{\text {total }}$ values in an SFSI system. For example, using PC projection with $L=16$, the minimum $T_{\text {total }}$ to achieve RMSE of 0.15 in the AFSI system is $0.34 \mathrm{~s}$, which is 2.57 times less than the minimum $T_{\text {total }}$ value $0.875 \mathrm{~s}$ in the SFIS system. We list all the minimum $T_{\text {total }}$ for both PC-based and Hadamardbased FSI systems for $L=1,4$, and 16 in Tables $\underline{3}$ and 4 , respectively. Note that the minimum $T_{\text {total }}$ values for PC-based and Hadamard-based FSI systems increases as $L$ increases due to the reduced measurement SNR for increasing $L$. Therefore, we can conclude that when measurement noise exists, the FSI system with $L=1$ parallel architecture presents the best performance. Hence, a DMD-based single detector FSI system implementation is used to support our simulation study of SFSI and AFSI systems [18].
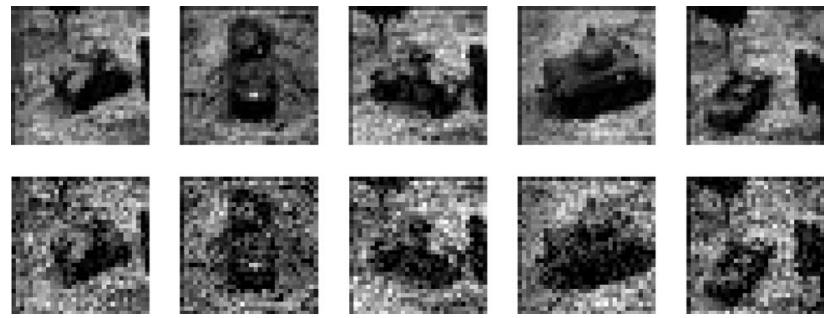

Fig. 8. Object examples (top row) and their noisy measurements (bottom row) when $\sigma^{2}=1 / T_{0}$ with $T_{0}=0.02 \mathrm{~s}$.

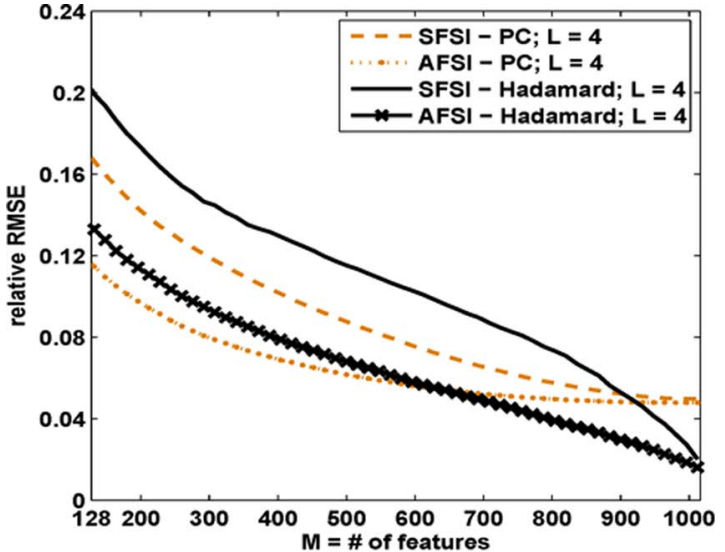

(a)

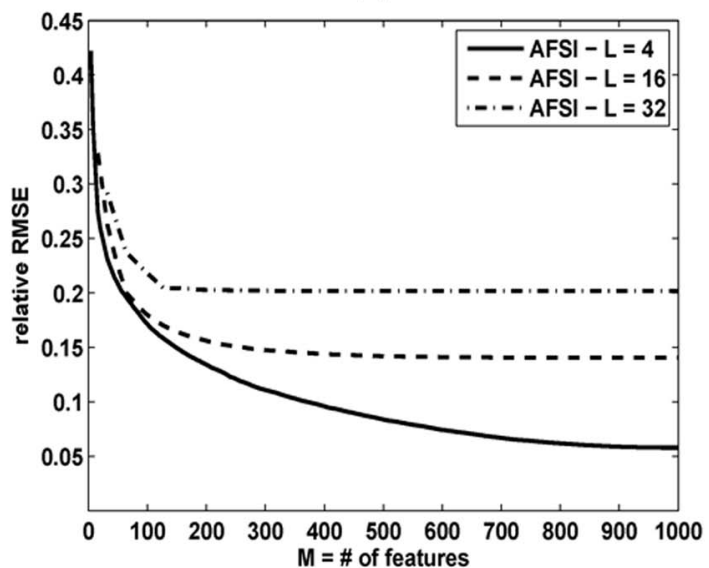

(b)

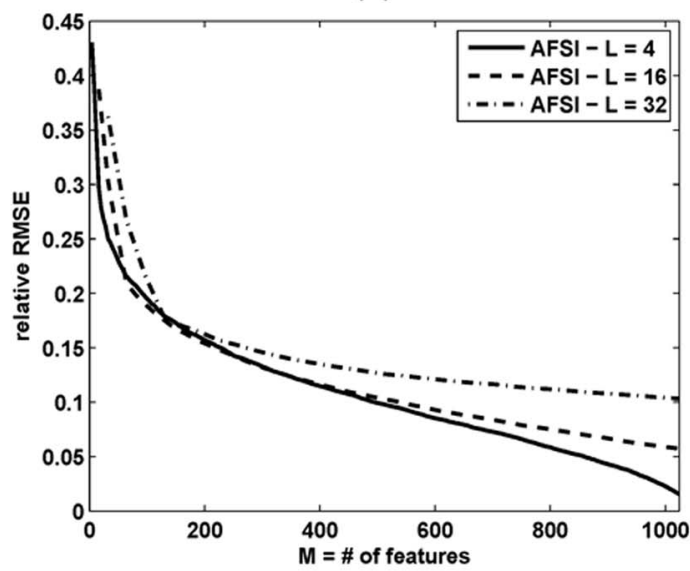

(c)

Fig. 9. (Color online) RMSE versus $M$ using (a) a PC and Hadamard AFSI system for $L=4$ when $T_{0}=0.02 \mathrm{~s}$, (b) PC, and (c) Hadamard AFSI system for different $L$ using high-diversity training set. 


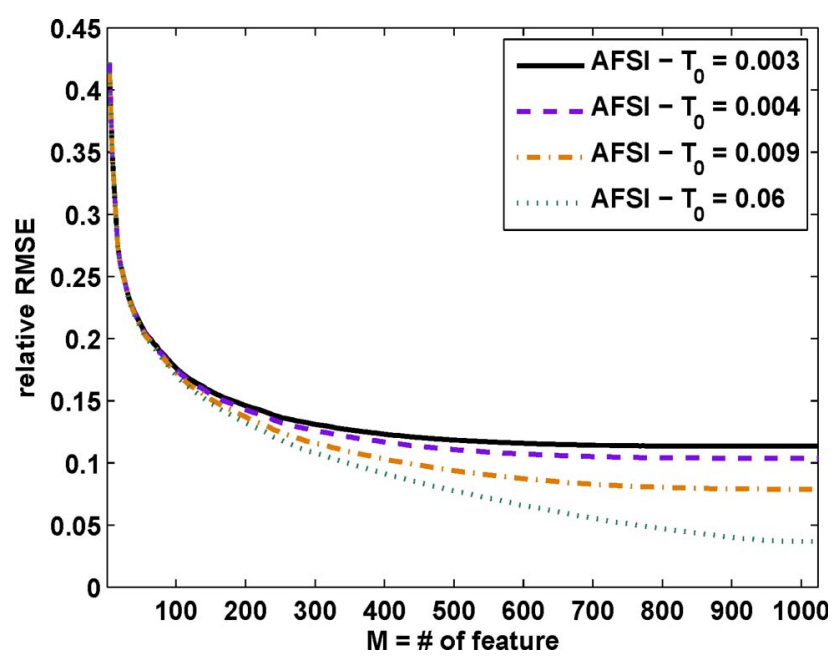

Fig. 10. (Color online) RMSE versus $M$ for different $T_{0}(s)$ in a PC-based AFSI system using a high-diversity training set when $L=32$

\section{Experiment}

Figure 12 shows a block diagram representation of the FSI imager experiment design. Here, a liquid crystal display (LCD) monitor is used to display an object of size $31.5 \mathrm{~mm} \times 31.5 \mathrm{~mm}$. An imaging lens images the object on to a 0.17 in HVGA DMD array (TI Pico projector [25]), which is controlled by a Beagle Board [26]. The DMD array modulates the image by switching its micromirrors between "on" and "off" states for a duration that is linearly proportional to each element of the projection vector. The modulated image reflected from the DMD is spatially integrated by the condensing lens onto a single detector that yields a noisy feature measurement corresponding to the projection vector displayed on the DMD. Subsequently, the signal from the detector is digitized by a National Instruments data acquisition (DAQ) system (NI USB-6251) for further processing. The LCD monitor and the NI DAQ system are controlled by a PC.

To quantify the reconstruction quality of the object estimate, we employ an SBIG Astronomical Instrument CCD camera (ST-2000XM) to measure a reference quality image of the object. Note that the PCA and Hadamard projection vectors contain positive and negative elements. However, the DMD array can display only positive values. Therefore, to implement the projection vector $\mathbf{f}$ with this positivity constraint, we divide it into positive and negative parts, $\mathbf{f}_{+}$and $\mathbf{f}_{-}$, respectively, where $\mathbf{f}_{+}=(\mathbf{f}+|\mathbf{f}|) / 2$ and $\mathbf{f}_{-}=(|\mathbf{f}|-\mathbf{f}) / 2$. To improve the light throughput, we normalize $\mathbf{f}_{+}$and $\mathbf{f}_{-}$with their maximum values $c_{+}$and $c_{-}$, respectively. After acquiring the two measurements $y_{+}=\mathbf{f}_{+}^{T} \mathbf{x} / c_{+}+n_{+}$and $y_{-}=\mathbf{f}_{-}^{T} \mathbf{x} / c_{-}+n_{-}$, where $n_{+}$and $n_{-}$are the measurement noise, we use following equation to estimate feature value $\mathbf{f}^{T} \mathbf{x}$ :

$$
y=c_{+} * y_{+}-c_{-} * y_{-}=\mathbf{f}^{T} \mathbf{x}+c_{+} * n_{+}-c_{-} * n_{-} .
$$

During the system calibration, we note that the DMD has a nonlinear response, as do other display devices, such as the LCD monitor. We linearize the DMD and monitor response by precompensating the input image data. A dark frame measurement is used to reduce the various biases in the experiment. Using these calibration methods, we are able to achieve measurements $y_{+}, y_{-}$for PCA and Hadamard projections, which are within $2 \%$ to $3 \%$ of their ideal values. We model this experiment error as Gaussian noise in our measurement error model for both SFSI and AFSI algorithms.

Recall that our simulation study demonstrated that with the parallel architecture, an AFSI system with $L=1$ achieves the best reconstruction performance with noisy measurements. Therefore, in the experiment, we choose the $L=1$ case to quantify the AFSI and SFSI system performance. Thus, accordingly in the experiment, we adapt the next projection vector after each feature measurement using the adaptation algorithms described earlier. In the following results, we use four face images, as shown in Fig. 13, for evaluating the system performance. For the adaptation algorithm, we employ the same high-diversity training set $A_{H}$ as the one used in the simulation study. Figure 14(a) shows the object estimates obtained using 128 PCA features from the SFSI and AFSI systems in the upper and the lower rows, respectively. Similarly, the object estimates based on 128 Hadamard features from the SFSI and AFSI systems are shown in Fig. 14(b). Note that the corresponding reconstruction RMSE value is labeled under each object estimate. First, we observe that the AFSI system achieves better reconstruction performance compared with the SFSI system in the case of both PCA and Hadamard projections as predicted by the simulation study. Note that the average RMSE values in the PC-based and Hadamard-based AFSI systems are 0.128 and 0.143 , respectively, compared to 0.163 and 0.179 for the SFSI system. This represents an RMSE improvement of $21.2 \%$ for PCA and $20.1 \%$ for Hadamard projections. The RMSE improvement is also visually noticeable in the AFSI system object estimates showing improved contrast and finer details compared to estimates obtained with the SFSI system. These RMSE results are summarized in Table 5. It is also worth noting that the reconstructions based on $\mathrm{PC}$ features have a smaller RMSE relative to the reconstructions derived from Hadamard features, which affirms the superior performance of the PCA measurement basis as predicted by simulation study. In fact, even with 160 Hadamard features, the resulting object estimate, shown in Fig. 15, is still slightly worse than that obtained using $\overline{128}$ PCA features.

Note that the performance improvement with the AFSI system was obtained for face objects in the experiment while the simulation study had used tank objects. Therefore, in order to assess the quality of the experimental results, it is important to compare it to the simulated performance of AFSI and SFSI systems for these objects. Table 6 presents the RMSE results for both PC and Hadamard-based AFSI and 


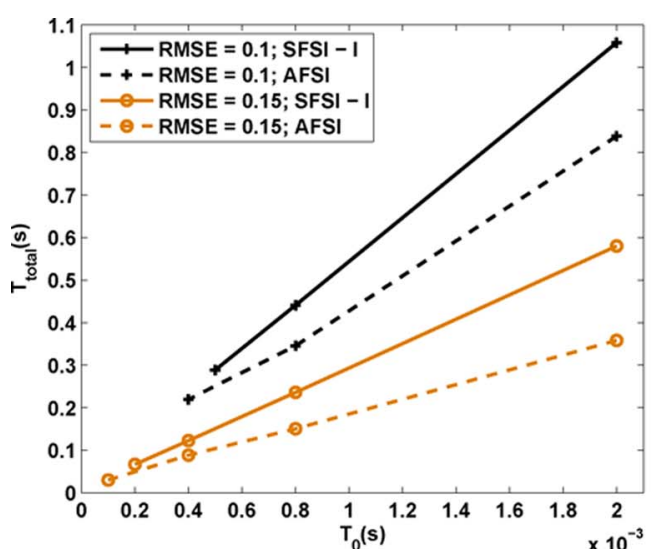

(a)

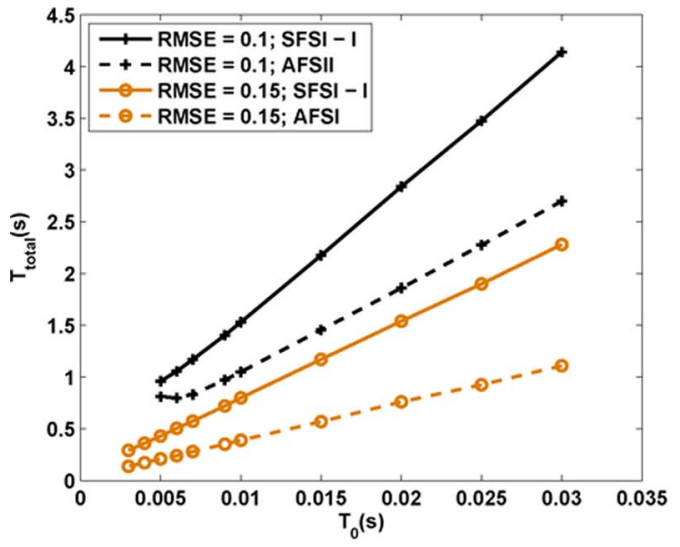

(b)

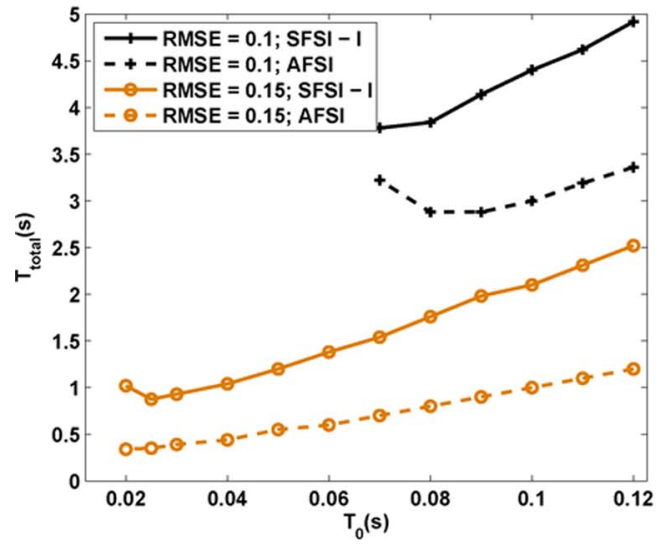

(c)

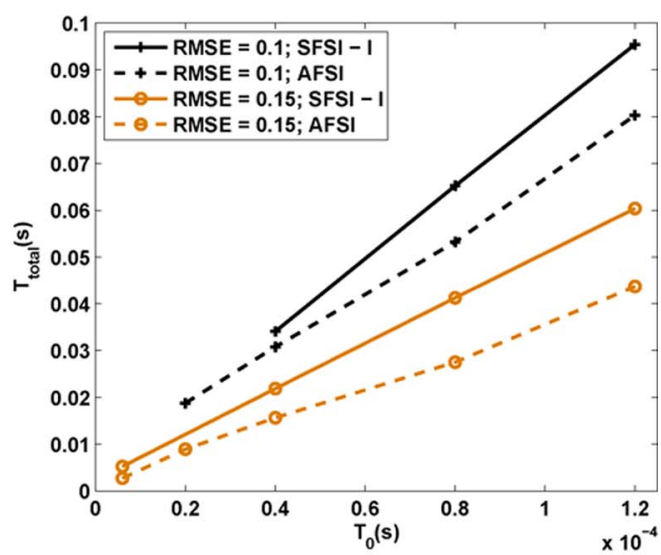

(d)

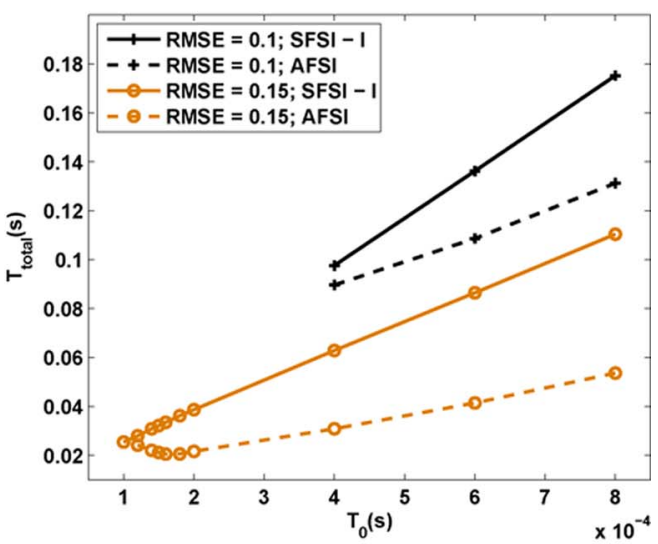

(e)

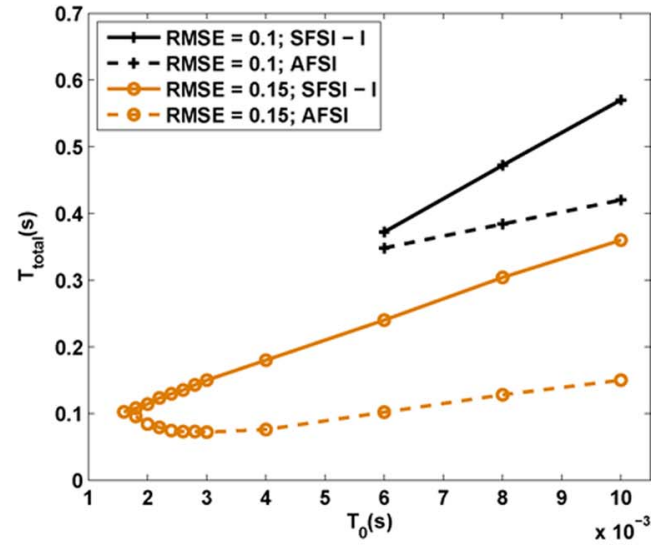

(f)

Fig. 11. (Color online) $T_{\text {total }}$ versus $T_{0}$ for (a) $L=1$, (b) $L=4$, and (c) $L=16$, PC-based; and (d) $L=1$, (e) $L=4$, and (f) $L=16$, Hadamardbased SFSI and AFSI systems using a high-diversity training set.

Table 3. Minimum $T_{\text {total }}$ in PC-based SFSI and AFSI Systems for $L=1,4$, and 16 Using $A_{H}$

\begin{tabular}{ccccccc}
\hline & & \multicolumn{2}{c}{ SFSI } & & \multicolumn{2}{c}{ AFSI } \\
\cline { 3 - 4 } \cline { 6 - 7 }$L$ & $R M S E$ & $T_{0}(\mathrm{~ms})$ & $T_{\text {total }}(\mathrm{s})$ & & $T_{0}(\mathrm{~ms})$ & $T_{\text {total }}(\mathrm{s})$ \\
\hline \multirow{2}{*}{1} & 0.10 & 0.4 & 0.2884 & & 0.4 & 0.2192 \\
& 0.15 & 0.1 & 0.0668 & & 0.1 & 0.03 \\
\multirow{2}{*}{4} & 0.10 & 5 & 0.96 & & 6 & 0.798 \\
& 0.15 & 3 & 0.291 & & 3 & 0.138 \\
\multirow{2}{*}{16} & 0.10 & 70 & 3.78 & & 80 & 2.88 \\
& 0.15 & 25 & 0.875 & & 20 & 0.34 \\
\hline
\end{tabular}

Table 4. Minimum $T_{\text {total }}$ in Hadamard-Based SFSI and AFSI Systems for $L=1,4$, and 16 Using $A_{H}$

\begin{tabular}{cccclll}
\hline & & \multicolumn{2}{c}{ SFSI } & & \multicolumn{2}{c}{ AFSI } \\
\cline { 3 - 4 } \cline { 6 - 7 }$L$ & $R M S E$ & $T_{0}(\mathrm{~ms})$ & $T_{\text {total }}(\mathrm{s})$ & & $T_{0}(\mathrm{~ms})$ & $T_{\text {total }}(\mathrm{s})$ \\
\hline \multirow{2}{*}{1} & 0.10 & 0.04 & 0.0341 & & 0.02 & 0.0188 \\
& 0.15 & 0.006 & 0.0052 & & 0.006 & 0.0027 \\
4 & 0.10 & 0.4 & 0.0912 & & 0.4 & 0.0728 \\
& 0.15 & 0.1 & 0.0254 & & 0.16 & 0.0205 \\
16 & 0.10 & 6 & 0.372 & & 6 & 0.348 \\
& 0.15 & 1.6 & 0.1024 & & 2.6 & 0.0728 \\
\hline
\end{tabular}




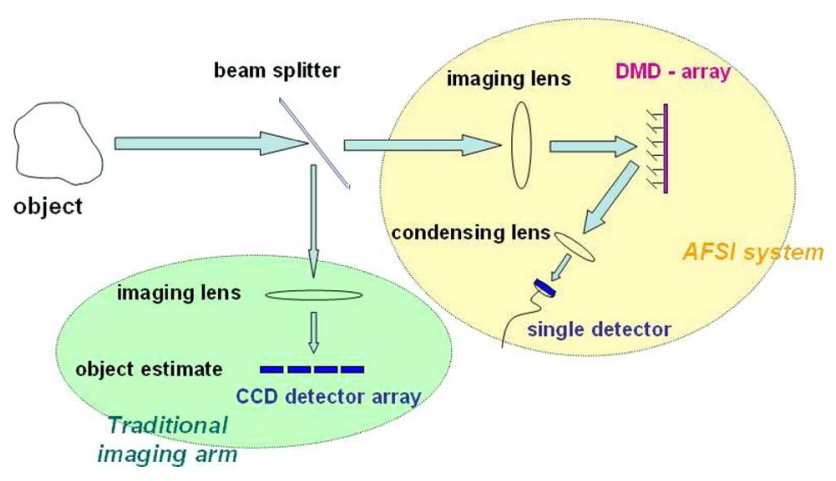

Fig. 12. (Color online) Experiment setup diagram.
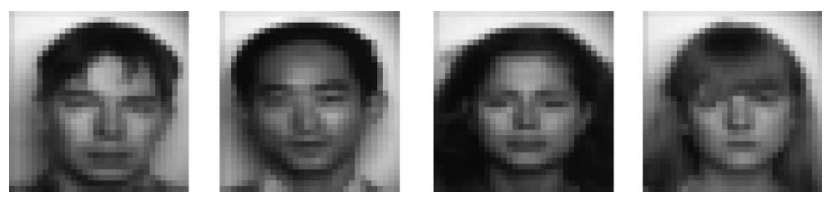

Fig. 13. Testing samples in experiment.

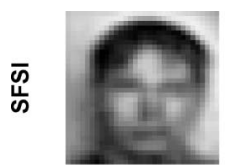

0.1625

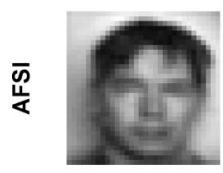

0.1200

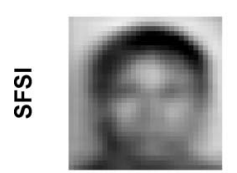

0.1953

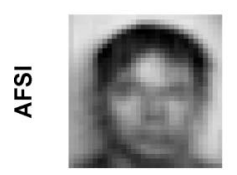

0.1373

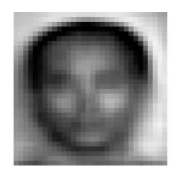

0.1728

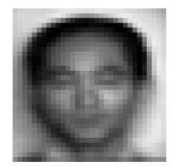

0.1429

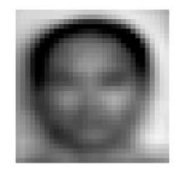

0.1818

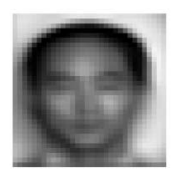

0.1544

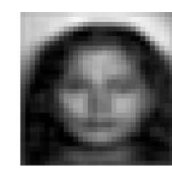

0.1648

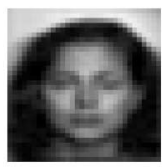

0.1350

(a)

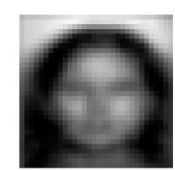

0.1698

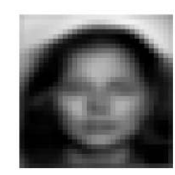

0.1559

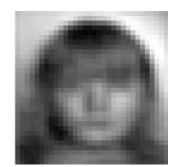

0.1510

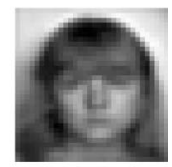

0.1155

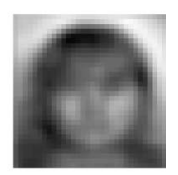

0.1615

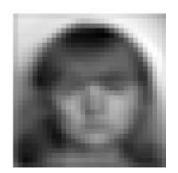

0.1265 (b)

Fig. 14. Reconstructions from (a) 128 PCA, and (b) 128 Hadamard experimental feature measurements in SFSI and AFSI systems for a $32 \times 32$ object when $L=1$.

Table 5. RMSE in Experimental SFSI and AFSI Systems for $L=1$ with $M=128$ Using High-Diversity Training Sets

\begin{tabular}{cccccc}
\hline & \multicolumn{2}{c}{ PCA } & & \multicolumn{2}{c}{ Hadamard } \\
\cline { 2 - 3 } \cline { 5 - 6 } & $\begin{array}{c}\text { Relative } \\
\text { RMSE }\end{array}$ & $\begin{array}{c}\text { RMSE } \\
\text { Improvement }\end{array}$ & & $\begin{array}{c}\text { Relative } \\
\text { RMSE }\end{array}$ & $\begin{array}{c}\text { RMSE } \\
\text { Improvement }\end{array}$ \\
\hline SFSI & 0.163 & $\times$ & & 0.179 & $\times$ \\
AFSI & 0.128 & $21.24 \%$ & & 0.143 & $20.11 \%$ \\
\hline
\end{tabular}

0.1726

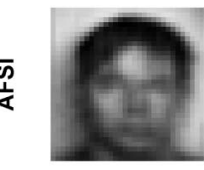

0.1342

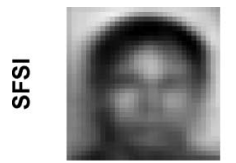

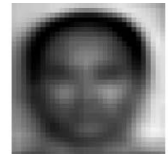

0.1773

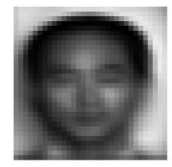

0.1540

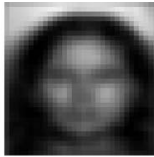

0.1691

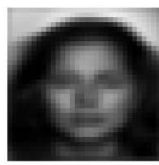

0.1513

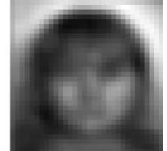

0.1582

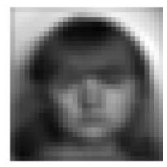

0.1256
Fig. 15. Reconstructions from 160 Hadamard experimental feature measurements in SFSI and AFSI systems when $L=1$.

SFSI systems obtained from the experiment and the simulation study for face and tank objects. Note that the noise variance used in the simulation study was based on the measurement error estimated from the experiment. From the RMSE results reported in the table, we can make the following observations. First, the RMSE values from the simulation study match well, to within a few percent, with the actual RMSE values obtained in the experiment. One reason for the small deviations between the experimental and the simulation RMSE values is that the simulation does not accurately model all the nonidealities typically encountered in the experiment. These include spatial nonuniformity of the DMD array, residual nonlinearity and bias in the DMD and the detector response after calibration, and temporal fluctuations in the object irradiance during the sequential data measurement process. Second, the simulation results for face objects, like the tank objects, show that the PC-based AFSI and SFSI systems yield better RMSE performance compared to Hadamard-based FSI imagers. It is also interesting to observe that the performance improvement between AFSI and SFSI systems for face objects is smaller than that for tank objects, as shown by the simulation results summarized in Table 7. To understand this, let us consider the PC-based F SI systems. Figure 16 shows the first five PC projection vectors obtained from the initial high-diversity training set $A_{H}$, which includes face, tank, and building objects. Note that among all five projection vectors, the underlying structure resembles an average low-pass version of the face objects. This indicates that although the number of face training samples is only $1 / 3$ of the total number of samples in the training set, the spatial statistics of the face objects are the dominant factor in determining the low-order PC projection vectors. Thus, it is reasonable to interpret that these low-order $\mathrm{PC}$ vectors in SFSI systems already incorporate strong prior knowledge about face objects, as compared to, say, the tank objects. As a result, the adaptation advantage and the equivalent RMSE improvement obtained from the AFSI system is smaller for face objects than for tank objects, as the static PC projection vectors are already biased toward face objects. 
Table 6. RMSE for Face and Tank Reconstructions from $128 \mathrm{PC}$ and Hadamard Experimental, Simulated Feature Values

in SFSI and AFSI Systems for $L=1$ Using $A_{H}$

\begin{tabular}{|c|c|c|c|c|c|c|}
\hline & \multicolumn{3}{|c|}{ PCA } & \multicolumn{3}{|c|}{ Hadamard } \\
\hline & \multicolumn{2}{|c|}{ Face } & \multirow{2}{*}{$\frac{\text { Tank }}{\text { Simulation }}$} & \multicolumn{2}{|c|}{ Face } & \multirow{2}{*}{$\frac{\text { Tank }}{\text { Simulation }}$} \\
\hline & Experiment & Simulation & & Experiment & Simulation & \\
\hline SFSI & 0.163 & 0.158 & 0.203 & 0.179 & 0.174 & 0.234 \\
\hline AFSI & 0.128 & 0.122 & 0.135 & 0.143 & 0.142 & 0.164 \\
\hline
\end{tabular}

Table 7. System Performance Improvement in Terms of RMSE for Face and Tank Reconstructions from 128 PC and Hadamard Simulated Feature Values in SFSI and AFSI Systems for $L=1$ Using $A_{H}$

\begin{tabular}{ccccc}
\hline \multicolumn{2}{c}{ PCA } & & \multicolumn{2}{c}{ Hadamard } \\
\cline { 2 - 3 } Face & Tank & & Face & Tank \\
\hline $22.7 \%$ & $33.4 \%$ & & $18.4 \%$ & $29.9 \%$ \\
\hline
\end{tabular}
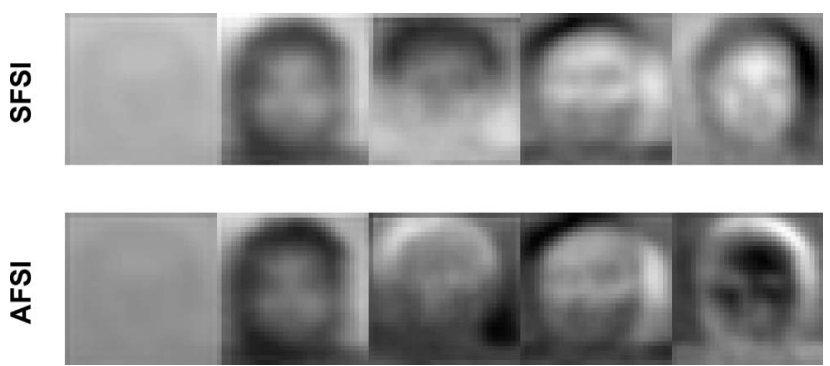

Fig. 16. First five PC vectors in SFSI and AFSI system for face object 1 when $L=1$.

\section{Conclusion}

In this paper, we analyzed an adaptive FSI system for a reconstruction task using PC and Hadamard projections. We employed a training set to design $\mathrm{PC}$ and Hadamard projections. The adaptation is achieved by updating the training set based on the collected feature measurements. We developed the algorithms for PC and Hadamard-based AFSI systems for noise-free and noisy measurements. Using a parallel architecture FSI system, we demonstrate that the AFSI system has superior performance compared to a SFSI system. The improvement of reconstruction error RMSE from SFSI system to AFSI system is about $30 \%$ for PC and Hadamard projections using tank-testing samples. We use a DMDbased experiment setup to validate the simulation results and achieved an average AFSI improvement larger than $20 \%$ using face objects. The experiment results support our simulation study and demonstrate the superior performance of AFSI relative to SFSI.

\section{References}

1. S. Park, M. Park, and M. Kang, "Super-resolution image reconstruction: A technical overview," IEEE Signal Process. Mag. 20, 21-36 (2003).
2. S. Prasad, "Digital super-resolution and the generalized sampling theorem," J. Opt. Soc. Am. A 24, 311-325 (2007).

3. M. Shankar, R. Willett, N. Pitsianis, T. Schulz, R. Gibbons, R. Te Kolste, J. Carriere, C. Chen, D. Prather, and D. Brady, "Thin infrared imaging systems through multichannel sampling," Appl. Opt. 47, B1-B10 (2008).

4. E. R. Dowski, Jr., and W. T. Cathey, "Extended depth of field through wave-front coding," Appl. Opt. 34, 1859-1866 (1995).

5. M. A. Neifeld and A. Ashok, "Pseudorandom phase masks for super-resolution imaging from subpixel shifting," Appl. Opt. 46, 2256-2268 (2007).

6. M. A. Neifeld and A. Ashok, "Point spread function engineering for iris recognition system design," Appl. Opt. 49, B26B39 (2010).

7. M. A. Neifeld and P. M. Shankar, "Feature specific imaging," Appl. Opt. 42, 3379-3389 (2003).

8. E. Candès, J. Romberg, and T. Tao, "Robust uncertainty principles: Exact signal reconstruction from highly incomplete frequency information," IEEE Trans. Inf. Theory 52, 489509 (2006).

9. D. Donoho, "Compressed sensing," IEEE Trans. Inf. Theory 52, 1289-1306 (2006).

10. L. Crocco, M. D’Urso, and T. Isernia, "Faithful nonlinear imaging from only-amplitude measurements of incident and total fields," Opt. Express 15, 3804-3815 (2007).

11. O. Bucci, L. Crocco, M. D’Urso, and T. Isernia, "Inverse scattering from phaseless measurements of the total field on open lines," J. Opt. Soc. Am. A 23, 2566-2577 (2006).

12. M. D’Urso, K. Belkebir, L. Crocco, T. Isernia, and A. Litman, "Phaseless imaging with experimental data: facts and challenges," J. Opt. Soc. Am. A 25, 271-281 (2008).

13. S. Agaian, Hadamard Matrices and Their Applications (Springer-Verlag, 1985).

14. Y. Tsaig and D. Donoho, "Extensions of compressed sensing," Signal Processing 86, 549-571 (2006).

15. E. J. Cand'es and M. B. Wakin, "An introduction to compressive sampling," IEEE Signal Process. Mag. 25, 21-30 (2008).

16. R. N. Jarvis Haupt, "Signal reconstruction from noisy random projections," IEEE Trans. Inf. Theory 52, 4036-4048 (2006).

17. D. Takhar, J. Laska, M. Wakin, M. Duarte, D. Baron, S. Sarvotham, K. Kelly, and R. Baraniuk, "A new compressive imaging camera architecture using optical-domain compression," Proc. SPIE 6065, 606509 (2006).

18. M. Duarte, M. Davenport, D. Takhar, J. Laska, T. Sun, K. Kelly, and R. Baraniuk, "Single-pixel imaging via compressive sampling," IEEE Signal Process. Mag. 25, 83-91 (2008).

19. J. Ke, P. M. Shankar, and M. A. Neifeld, "Distributed imaging using an array of compressive cameras," Opt. Commun. 282, 185-197 (2009).

20. P. K. Baheti and M. A. Neifeld, "Feature-specific structured imaging," Appl. Opt. 45, 7382-7391 (2006).

21. N. Pitsianis, D. Brady, A. Portnoy, X. Sun, T. Suleski, M. Fiddy, M. Feldman, and R. TeKolste, "Compressive imaging sensors," Proc. SPIE 6232, 62320A (2006). 
22. N. Pitsianis, D. Brady, and X. Sun, "Sensor-layer image compression based on the quantized cosine transform," Proc. SPIE 5817, 250-257 (2005).

23. J. Ke and M. A. Neifeld, "Optical architectures for compressive imaging," Appl. Opt. 46, 5293-5303 (2007).
24. I. Jolliffe, Principle Component Analysis (Springer, 2002).

25. Pico projector, http://focus.ti.com/dlpdmd/docs/dlpdiscovery tsp?sectionId $=60$ \&tabId $=2234$.

26. Beagle Board, http://beagleboard.org/. 